\title{
Reduced miRNA-214 expression in oral mucosa contributes to the pathogenesis of oral lichen planus by targeting CD44
}

\author{
HAIYAN ZHENG ${ }^{1}$ and SHAOHUI LI ${ }^{2}$ \\ ${ }^{1}$ Department of Dermatology, Jining First People's Hospital, Jining, Shandong 272011; \\ ${ }^{2}$ Department of Prosthodontics, Jining Hospital of Stomatology, Jining, Shandong 272001, P.R. China
}

Received December 13, 2015; Accepted April 4, 2017

DOI: $10.3892 / \mathrm{mmr} .2017 .8019$

\begin{abstract}
The present study aimed to investigate the role of miRNAs during the pathogenesis of oral lichen planus (OLP). OLP is a chronic inflammatory disorder, which involves T-cell mediated autoimmunity and affects the skin, scalp, nails and mucosa. Abundant T lymphocytes have been demonstrated to infiltrate the oral mucosa, in which the activated T cells trigger apoptosis of oral epithelial cells. Overexpression of osteopontin (OPN) and CD44 has been observed in the mucosa of patients with OLP, and it has been confirmed that OPN suppresses the apoptosis of activated $\mathrm{CD}^{+} \mathrm{T}$ cells via CD44. The present study initially detected the protein and mRNA expression of CD44 and OPN in the mucosa of patients with OLP by western blot analysis and reverse transcription-quantitative polymerase chain reaction (RT-qPCR), and confirmed the previously reported overexpression of CD44 and OPN in patients with OLP. The current study demonstrated, by RT-qPCR, that the expression of microRNA-214 (miR-214), miR-216a and miR-216b was significantly reduced in patients with OLP. By analyzing the association between the protein level of CD44 and the expression of microRNAs (miRNAs), the present study identified a negative correlation between the expression of miR-214 and CD44 in the mucosa samples of patients with OLP. Subsequently, the present study confirmed that miR-214 represses endogenous CD44 expression by targeting the 3'untranslated region in HeLa, Raji and Jurkat cells. The current study indicates that reduced miR-214 may be associated with OLP and, therefore, may be a candidate for drug development.
\end{abstract}

\section{Introduction}

Oral lichen planus (OLP) is a chronic inflammatory disorder in which T-cell mediated autoimmunity is implicated (1).

Correspondence to: Dr Shaohui Li, Department of Prosthodontics, Jining Hospital of Stomatology, 64 Gongqingtuan Road, Jining, Shandong 272001, P.R. China

E-mail: shaohui_li81@163.com

Key words: oral lichen planus, microRNA, CD44, osteopontin
OLP affects $0.5-2 \%$ of the population of China and disease onset occurs between 30 and 60 years of age (2). Although the etiology of OLP remains unclear, genetic predisposition, autoimmunity and viral infection are reported to be associated with OLP (3).

During inflammation, osteopontin (OPN) is primarily expressed by immunocytes, including natural killer cells, activated T cells and macrophages (4). CD44 is a receptor for OPN and is associated with the mediation of cell chemotaxis and attachment (5). It has been demonstrated that OPN is overexpressed in various types of autoimmune diseases (6-8). Additionally, there are reports that OPN is overexpressed in the serum and oral epithelia of patients with OLP, which indicates a potential role for OPN in the pathogenesis of $\operatorname{OLP}(9,10)$.

MicroRNAs (miRNA) are a group of endogenous, short non-coding RNAs, which regulate gene expression through targeting the 3'untranslated region (UTR) of mRNA. miRNAs have been identified in various organisms and many of them are evolutionary conserved. Additionally, it is estimated that $>50 \%$ of all human protein-coding genes are potentially regulated by miRNAs (11). It has been previously reported that the miRNA profile was altered in the oral mucosa of patients with OLP (12). However, the effect of altered miRNA levels in OLP requires further investigation.

In the present study, 11 miRNAs that may target CD44 directly were selected. The expression levels of the selected miRNAs in oral mucosa samples from 33 patients and 33 controls were detected. In addition, the expression of the CD44 protein was examined by western blot analysis. The results of the present study demonstrated that two miRNAs were significantly reduced in OLP samples compared with controls and the expression of CD44 was upregulated in OLP samples. Subsequently, correlation analysis was performed to assess the correlation between the expression of miRNAs and CD44 in vitro and in patients with OLP.

\section{Materials and methods}

Participants and sample collection. Oral mucosa samples were collected during incisional biopsy procedures following surgical removal from 33 patients (age, 23-37 years; mean age, 38.7 years; 18 female; 15 male), who were recruited from Jining First People's Hospital (Jining, China) between February 2013 and November 2014. Following collection, one 
half of the specimens were examined at the pathology department of Jining Hospital (Jining, China) to verify the diagnosis of OLP. The other half were directly frozen in liquid nitrogen and were stored at $-80^{\circ} \mathrm{C}$ until further analysis. The control group was composed of tissue samples from elective oral surgery whereby a histopathological examination excluded any diseases of the oral mucosa. The study was approved by the ethics board of Jining hospital of Stomatology (Jining, China). All participants were informed by a patient information sheet about the intention of the study and written informed consent according to the Helsinki convention was obtained from all participants.

Cell culture. HeLa human cervical carcinoma cells (National Infrastructure of Cell Line Resource, Beijing, China) were maintained in Dulbecco's modified Eagle's medium (HyClone; GE Healthcare Life Sciences, Logan, UT, USA) supplemented with $10 \%$ fetal calf serum (FCS; HyClone; GE Healthcare Life Sciences) and $50 \mathrm{U} / \mathrm{ml}$ penicillin and $0.1 \mathrm{mg} / \mathrm{ml}$ streptomycin (HyClone; GE Healthcare Life Sciences) at $37^{\circ} \mathrm{C}$ in a humidified atmosphere containing $5 \% \mathrm{CO}_{2}$. Raji Burkitt lymphoma B-cell lines and Jurkat T-lymphocytes were obtained from the National Infrastructure of Cell Line Resources, and were cultured in RPMI 1640 medium (HyClone; GE Healthcare Life Sciences) supplemented with $10 \%$ heat-inactivated FCS (Hyclone; GE Healthcare Life Sciences), $100 \mathrm{U} / \mathrm{ml}$ penicillin, $0.1 \mathrm{mg} / \mathrm{ml}$ streptomycin, $2 \mathrm{mM}$ L-glutamine, and $1 \mathrm{mM}$ sodium pyruvate at $37^{\circ} \mathrm{C}$ in a $5 \% \mathrm{CO}_{2}$ atmosphere.

RNA extraction and reverse transcription-quantitative polymerase chain reaction $(R T-q P C R)$. Prior to RT-qPCR, TargetScan (www.targetscan.org) was used to predict miRNAs that may target the 3'UTR of CD44, and 11 miRNAs were selected for RT-qPCR analysis. Total RNA was extracted from tissue samples by using TRIzol ${ }^{\circledR}$ reagent (Invitrogen; Thermo Fisher Scientific, Inc., Waltham, MA, USA) according to the manufacturer's protocol. The expression of miRNAs was detected by TaqMan miRNA RT-qPCR. Single-stranded cDNA was synthesized by using TaqMan MicroRNA Reverse Transcription kit (Applied Biosystems; Thermo Fisher Scientific, Inc.) at $16^{\circ} \mathrm{C}$ for $30 \mathrm{~min}$, followed by $42^{\circ} \mathrm{C}$ for $30 \mathrm{~min}$ and $85^{\circ} \mathrm{C}$ for $5 \mathrm{~min}$. cDNA was subsequently amplified by using TaqMan Universal PCR Master Mix (Applied Biosystems; Thermo Fisher Scientific, Inc.) together with miRNA-specific TaqMan MGB probes (Applied Biosystems; Thermo Fisher Scientific, Inc.). The primer sequences for OPN were: OPN forward, 5'-CATATGATGGCCGAGGTG ATAG-3' and OPN reverse 5'-AGGTGATGTCCTCGTCTG TA-3'. The primer sequences for CD44 were: CD44 forward 5'-GATGTCACAGGTGGAAGAAGAG-3' and CD44 reverse 5'-TTCCTTCGTGTGTGGGTAATG-3'. The catalog numbers for the miRNA probes were: MiR-135a, 000460; miR-135b, 002261; miR-143-3p, 002249; miR-199a, 002304; miR-204, 000508; miR-211, 477502_mat; miR-214, 000517; miR-216a, 002220; miR-216b, 002326; miR-320a, 002277; and miR-489, 466,550_mat. The thermocycling conditions were as follows: $95^{\circ} \mathrm{C}$ for $10 \mathrm{~min}$, followed by 40 cycles of $95^{\circ} \mathrm{C}$ for $15 \mathrm{sec}$ and $60^{\circ} \mathrm{C}$ for $1 \mathrm{~min}$ (ABI7500; Applied Biosystems; Thermo Fisher Scientific, Inc.). Results were normalized using the $\Delta \Delta \mathrm{Cq}$ method and U6 snRNA was used as internal control (13).
Each sample in each group was measured in triplicate and the experiment was repeated at least three times. The negative control was reacted with all PCR reagents except the reverse transcriptase.

Dual luciferase assay. A segment of 722 bp CD44 3'UTR containing 2 predicted miR-214 target regions was cloned into pmirGLO vector between $X b a \mathrm{I}$ and $X h o I$ sites, downstream of the firefly luciferase coding region (Promega Corporation, Madison, WI, USA) to generate luciferase reporter vectors. Mutant CD44 3'UTR was directly amplified using primers with 3 mutated nucleotides, and then cloned into the pmirGLO vector to generate the mutant reporter vector. For luciferase reporter assays, HeLa cells were seeded in 48-well plates $\left(5 \times 10^{4}\right.$ cells/well). miRNA mimics or inhibitors, or short nucleotide oligo controls, and luciferase reporter vectors, were co-transfected into HeLa cells by Lipofectamine ${ }^{\circledR} 2000$ (Invitrogen; Thermo Fisher Scientific, Inc.) at $37^{\circ} \mathrm{C}$ for $6 \mathrm{~h}$. At 2 days post-transfection, cells were harvested and assayed with the Dual-Luciferase ${ }^{\circledR}$ Reporter Assay System (Promega Corporation). Each treatment was performed in triplicate in 3 independent experiments. The results were expressed as relative luciferase activity (Firefly luciferase/Renilla luciferase). The sequence of the 2'-O-methoxyethyl (OMe) modified double strand miR-214 mimic is 5'-ACAGCAGGCACAGAC AGGCAGU-3' and the sequence of the 2'-OMe modified single strand miR-214 inhibitor is 5'-ACUGCCUGUCUGUGCCUG CUGU-3'. Scrambled 2'-OMe-modified RNA (5'-AAGGCA AGCUGACCCUGAAGU-3') was used as a negative control.

Western blot analysis. Protein was extracted from cells and tissue samples using radioimmunoprecipitation assay (RIPA) buffer (Thermo Fisher Scientific, Inc.), following the manufacturer's protocol. Cells were washed with cold PBS twice and incubated with cold RIPA buffer on ice for $5 \mathrm{~min}$. Samples were centrifuged at $\sim 14,000 \mathrm{x} \mathrm{g}$ at $4^{\circ} \mathrm{C}$ for $15 \mathrm{~min}$ to pellet the cell debris and the supernatant was transferred to a new tube. For tissue samples, the tissue was dissected with clean tools on ice and ice-cold RIPA buffer was added rapidly to the tube, followed by homogenizing with an electric homogenizer. Samples were centrifuged for $20 \mathrm{~min}$ at $15,000 \mathrm{xg}$ at $4^{\circ} \mathrm{C}$ and the supernatant was transferred to a new tube. The protein concentration was determined using a Bicinchoninic Acid Protein Assay kit (Thermo Fisher Scientific, Inc.). Protein extracts were boiled in SDS/ $\beta$-mercaptoethanol sample buffer (Sigma-Aldrich; Merck KGaA, Darmstadt, Germany) and $10 \mu \mathrm{g}$ protein from tissue or $20 \mu \mathrm{g}$ from cells were loaded into each lane of $8 \%$ polyacrylamide gels. The proteins were separated by electrophoresis, and the proteins in the gels were blotted onto polyvinylidene fluoride membranes (GE Healthcare Life Sciences, Chalfont, UK) by electrophoretic transfer. Membranes were subsequently incubated with rabbit anti-CD44 polyclonal antibody (cat. no. ab51037, 1:1,000 dilution; Abcam, Cambridge, MA, USA), rabbit anti-osteopontin polyclonal antibody (cat. no. ab8448, 1:1,000 dilution; Abcam) or mouse anti- $\beta$-actin monoclonal antibody (cat. no. sc-47778, 1:5,000 dilution; Santa Cruz Biotechnology Inc., Dallas, TX, USA) for $1 \mathrm{~h}$ at $37^{\circ} \mathrm{C}$. The specific protein-antibody complex was detected by using horseradish peroxidase-conjugated goat anti-rabbit (cat. no. ab6721, 1:5,000 dilution; Abcam) or 
rabbit anti-mouse (cat. no. ab6728, 1:5000 dilution; Abcam) antibody by incubation at $37^{\circ} \mathrm{C}$ for $2 \mathrm{~h}$. Detection by chemiluminescence was performed using the Pierce ${ }^{\mathrm{TM}}$ ECL Western Blotting Substrate (Thermo Fisher Scientific, Inc.), according to the manufacturer's protocol. The $\beta$-actin signal was used as a loading control. Densitometric analysis was performed using Quantity One software version 4.6.2 (Bio-Rad Laboratories, Inc. Hercules, CA, USA.) Results are exhibited as the mean \pm standard deviation of 2 replicates.

Histological analysis. The cases were classified histopathologically following the diagnostic criteria by van der Waal et al (14). All cases and controls underwent a hematoxylin and eosin standard histological procedure (15). Results were analyzed by $\chi^{2}$ analysis.

Statistical analysis. Data were analyzed by using SPSS Statistical Package version 15 (SPSS, Inc., Chicago, IL, USA). The correlation between miRNAs level and relative CD44 expression were analyzed by Spearman correlation analysis. Independent two group analyses were conducted using unpaired Student's t-test. Associations between the expression of miR-214, OPN or CD44 and histopathological features were analyzed by $\chi^{2}$-analysis. $\mathrm{P}<0.05$ was considered to indicate a statistically significant difference.

\section{Results}

OPN and CD44 mRNA and protein levels increase in patients with OLP. As OPN and CD44 have important roles during the pathogenesis of OLP, the present study initially detected the expression of OPN and CD44 in mucosa samples of patients with OLP and healthy controls. As presented in Fig. 1A, the mRNA expression levels of OPN and CD44 were significantly upregulated in patients with OLP, compared with controls $(\mathrm{P}<0.05)$. Protein levels of OPN and CD44 were also examined by western blot analysis. As demonstrated in Fig. 1B, protein expression of OPN $(\mathrm{P}<0.05)$ and $\mathrm{CD} 44(\mathrm{P}<0.01)$ was markedly increased in patients with OLP compared with controls. However, the protein expression of CD44 increased to a greater degree compared with increases in CD44 mRNA expression, indicating that post-transcriptional regulatory factors may contribute to the increased expression of CD44.

miR-214, miR-216a and miR-216b expression decreases in patients with OLP. As an important negative regulator of expression, miRNAs regulate the expression of target genes by directly targeting 3 'UTRs through post-transcriptional methods. To identify the miRNAs that repress the expression of CD44 and may be associated with the pathogenesis of OLP, miRNAs that target the CD44 3'UTR were predicted by using the following online bioinformatics tool: TargetScan (www.targetscan.org). A total of 11 miRNAs were selected, with target sites conserved among mammals. The expression levels of the selected candidate miRNAs were detected in the tissue samples of patients with OLP and controls by RT-qPCR. As presented in Fig. 2, the expression of miR-214, miR-216a and miR-216b was reduced significantly in patients with OLP compared with controls $(\mathrm{P}=0.031, \mathrm{P}=0.0060$ and $\mathrm{P}=0.0058$, respectively).
CD44 exhibits a negative correlation with miR-214. To investigate the association between the expression of CD44 and the 3 miRNAs that exhibited reduced expression in patients with OLP, the CD44 band density results presented in Fig. 1B were used for further analysis, to assess the correlation between the expression of CD44 and miRNAs. As presented in Fig. 3, the expression of miR-214 was negatively correlated with the protein level of CD44 $(\mathrm{P}=0.00030 ; \mathrm{r}=-0.44)$, indicating that reduced miR-214 may contribute to the increased expression of CD44 observed in patients with OLP compared with controls.

miR-214 suppresses luciferase activity in cell lines. To confirm whether CD44 is a direct target of miR-214, a luciferase activity reporter vector was constructed, attaching the 3'UTR of CD44 to the end of the firefly luciferase coding region (Fig. 4A and B). HeLa cells were transfected with miR-214 mimic or inhibitor and reporter vector. At $48 \mathrm{~h}$ post-transfection, cells were lysed and the luciferase activities were detected. As presented in Fig. 4C, firefly luciferase activity was reduced significantly by miR-214 mimic, compared with miR control group, and increased by miR-214 inhibitor addition compared with anti-miR control group. Furthermore, when 6 nucleotides were mutated, the luciferase activity was not repressed by miR-214 (Fig. 4D). The results indicated that miR-214 repressed luciferase activity by targeting the CD44 3'UTR.

Expression of CD44 in HeLa, Raji and Jurkat cell lines varies with transfection with miR-214 mimic or inhibitor. To further understand whether endogenous CD44 was repressed by miR-214, HeLa, Raji and Jurkat cells were transfected with miR-214 mimic or inhibitor. At $48 \mathrm{~h}$ post-transfection, cells were lysed and the expression of CD44 was detected by western blot analysis. As demonstrated in Fig. 5, the expression of endogenous CD44 was significantly reduced by miR-214 mimic compared with miR control group $(\mathrm{P}<0.05)$ and significantly increased by miR-214 inhibitor compared with anti-miR control group $(\mathrm{P}<0.05)$ in HeLa, Raji and Jurkat cells. The results indicate that CD44 is a direct target of miR-214.

Histological analysis. The histopathological features of patients with downregulated miR-214 were analyzed and it was observed that those with downregulated miR-214 had a significantly greater probability of exhibiting parakeratosis, lengthened epithelial crests and deep inflammatory infiltrates (Table I). Furthermore, overexpression of CD44 was associated with basal degeneration and deep inflammatory infiltrate (Table I). Overexpressed OPN was associated with basal degeneration, superficial inflammatory infiltrate and deep inflammatory infiltrate (Table I).

\section{Discussion}

CD44 is a transmembrane glycoprotein that acts as a receptor for a wide variety of extracellular matrix ligands, including hyaluronan, collagen, fibronectin, laminin and OPN. CD44 and receptor tyrosine kinases have been previously demonstrated to promote cell survival in a co-operative manner (16). CD44 is one of the characterized receptors of OPN that mediate the chemotaxis and attachment of immune cells. In OLP, abundant $\mathrm{T}$ lymphocytes have been previously demonstrated 
Table I. Association between the major histopathological features and the expression of miR-214, CD44 and OPN.

\begin{tabular}{|c|c|c|c|c|c|c|c|c|c|}
\hline \multirow[b]{2}{*}{ Histopathological feature } & \multicolumn{3}{|c|}{ miR-214 downregulation } & \multicolumn{3}{|c|}{ CD44 overexpression } & \multicolumn{3}{|c|}{ OPN overexpression } \\
\hline & No $(\%)$ & Yes $(\%)$ & P-value & No $(\%)$ & Yes $(\%)$ & P-value & No $(\%)$ & Yes $(\%)$ & P-value \\
\hline Parakeratosis & $2(18.2)$ & $14(63.6)$ & 0.014 & $3(33.3)$ & $13(54.2)$ & $>0.05$ & $4(50)$ & $12(48)$ & $>0.05$ \\
\hline Orthokeratosis & $9(81.2)$ & $8(36.4)$ & 0.014 & $6(66.7)$ & $11(45.8)$ & & $4(50)$ & $13(52)$ & $>0.05$ \\
\hline Hyperkeratosis & $5(45.5)$ & $9(40.9)$ & $>0.05$ & $4(44.4)$ & $10(41.7)$ & $>0.05$ & $3(37.5)$ & $11(44)$ & $>0.05$ \\
\hline atrophy & $3(27.3)$ & $7(31.8)$ & $>0.05$ & $2(22.2)$ & $8(33.3)$ & $>0.05$ & $3(37.5)$ & $7(28)$ & $>0.05$ \\
\hline Lengthened epithelial crest & $1(9.1)$ & $12(54.5)$ & 0.012 & $2(22.2)$ & $15(62.5)$ & 0.020 & $2(25)$ & $11(44)$ & $>0.05$ \\
\hline Basal degeneration & $9(81.8)$ & $19(86.4)$ & $>0.05$ & $6(66.7)$ & $22(91.7)$ & 0.00050 & $4(50)$ & $24(96)$ & 0.0016 \\
\hline Dyskeratosis & $7(63.6)$ & $15(68.2)$ & $>0.05$ & $5(55.6)$ & $17(70.8)$ & $>0.05$ & $5(62.5)$ & $17(68)$ & $>0.05$ \\
\hline Inflammatory infiltrate & $5(45.5)$ & $17(77.3)$ & $>0.05$ & $4(44.4)$ & $18(75)$ & 0.037 & $3(37.5)$ & $19(76)$ & 0.0074 \\
\hline Lymphocytes & $3(27.3)$ & $15(68.2)$ & $>0.05$ & $4(44.4)$ & $14(58.3)$ & $>0.05$ & $4(50)$ & $14(56)$ & $>0.05$ \\
\hline Plasmocytes & $2(18.2)$ & $8(36.4)$ & $>0.05$ & $3(33.3)$ & $7(29.2)$ & $>0.05$ & $2(25)$ & $8(32)$ & $>0.05$ \\
\hline Band-like inflammatory infiltrate & $4(36.4)$ & $14(63.6)$ & $>0.05$ & $3(33.3)$ & $15(62.5)$ & $>0.05$ & $2(25)$ & $16(64)$ & $>0.05$ \\
\hline Perivascular inflammatory infiltrate & $1(9.1)$ & $3(13.6)$ & $>0.05$ & $1(11.1)$ & $3(12.5)$ & $>0.05$ & $0(0)$ & $4(16)$ & $>0.05$ \\
\hline Superficial inflammatory infiltrate & $3(27.3)$ & $13(59.1)$ & $>0.05$ & $2(22.2)$ & $14(58.3)$ & $>0.05$ & $1(12.5)$ & $15(60)$ & 0.019 \\
\hline Deep inflammatory infiltrate & $3(27.3)$ & $15(68.2)$ & 0.026 & $1(11.1)$ & $17(70.8)$ & 0.0022 & $2(25)$ & $16(64)$ & 0.019 \\
\hline
\end{tabular}

Among the 33 patients, there were 22 patients with reduced miR-214 expression, 24 patients with overex pressed CD44 and 25 patients with overexpressed OPN. $\chi^{2}$-analysis was used to perform this analysis using SPSS statistical package version 15. miR-214, microRNA-214; OPN, osteopontin.
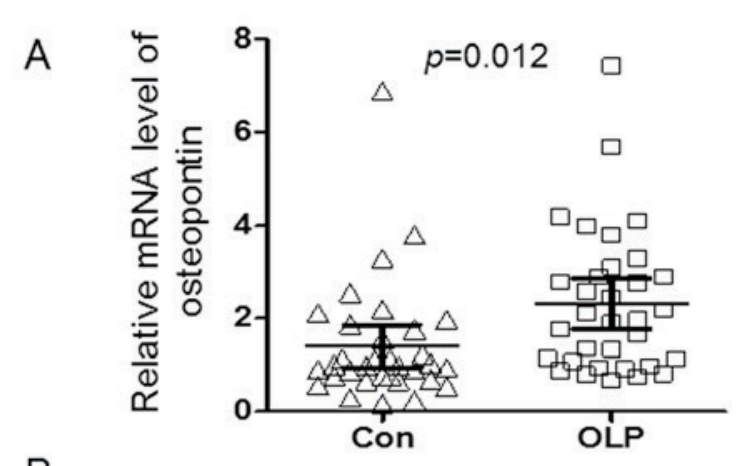

B
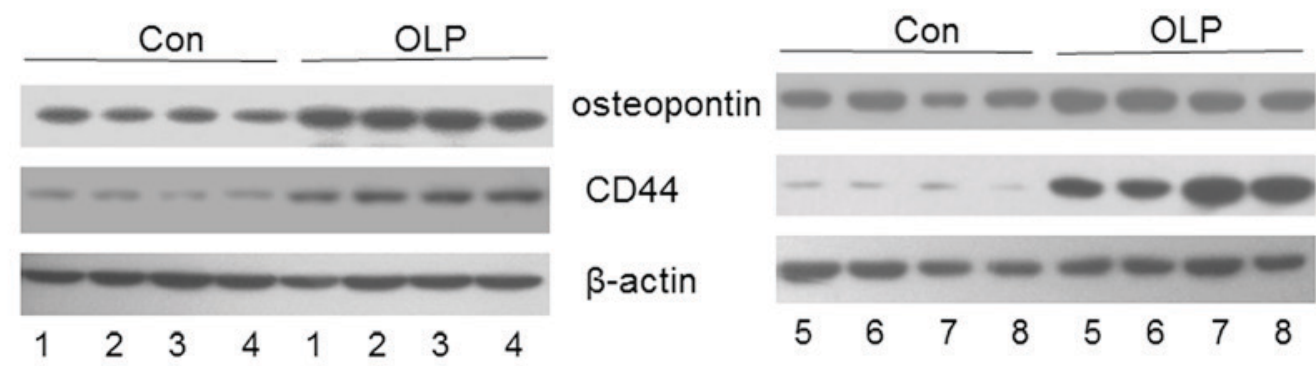

CD44

$\beta$-actin
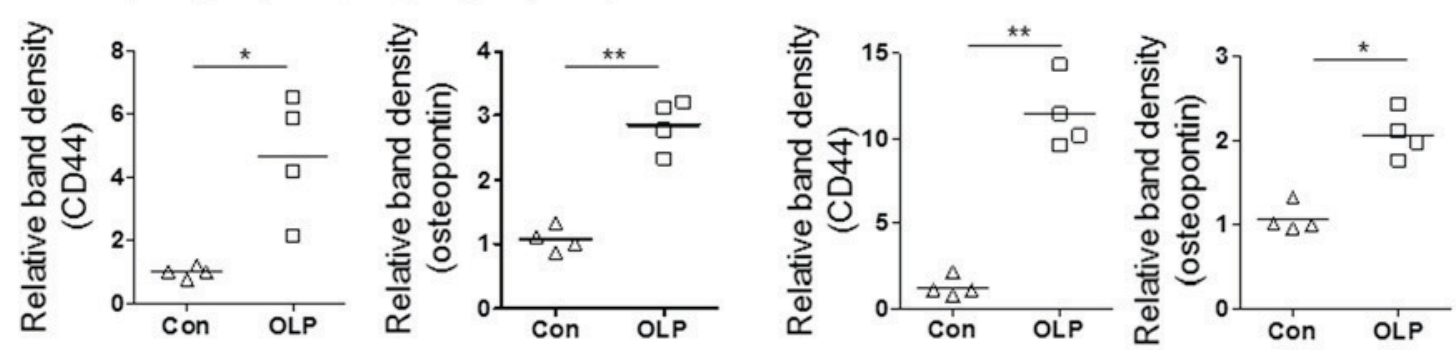

Figure 1. OPN and CD44 overexpression in patients with OLP. (A) mRNA expression levels of OPN and CD44 was detected by reverse transcription-quantitative polymerase chain reaction. (B) The expression of OPN and CD44 was detected by western blot analysis. Western blots were performed in duplicate. Data are presented as the mean \pm standard deviation. ${ }^{*} \mathrm{P}<0.05$ and ${ }^{* *} \mathrm{P}<0.01$. OPN, osteopontin; OLP, oral lichen planus; Con, control. 


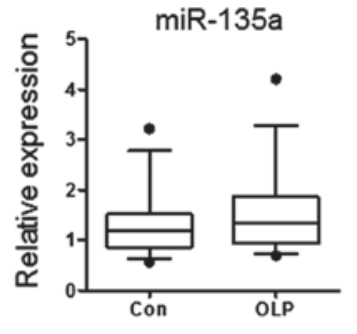

miR-204

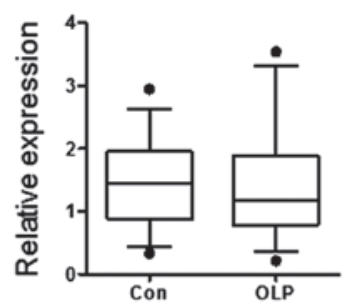

$\mathrm{miR}-216 \mathrm{~b}$

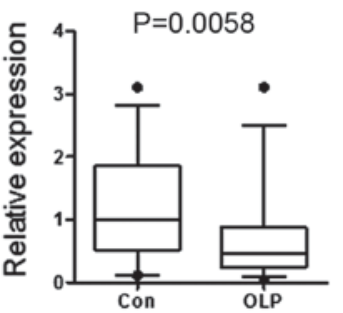

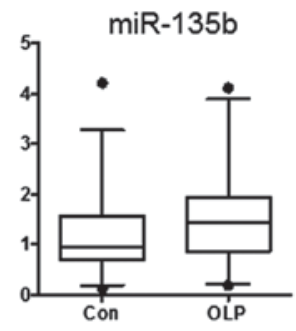

miR-211

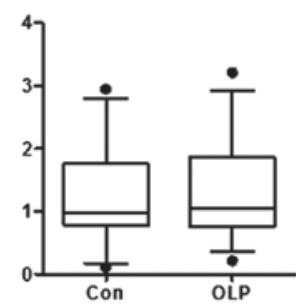

miR-302a

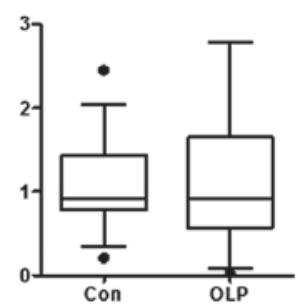

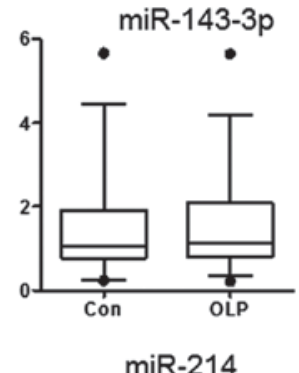

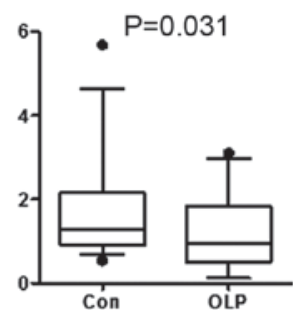

$\operatorname{miR}-489$

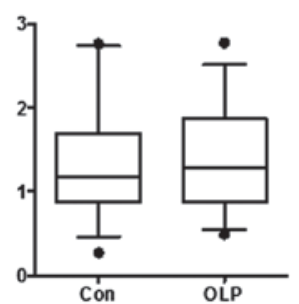

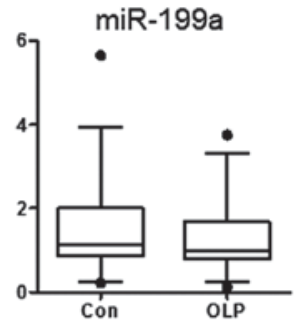

miR-216a

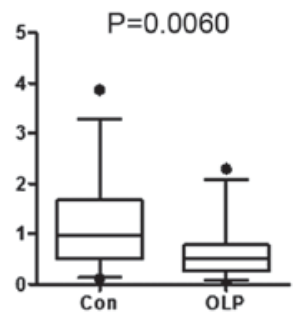

Figure 2. Reduced expression of miR-214, miR-216a and miR-216b in patients with OLP. Total RNA was extracted from the mucosa samples of 33 patients with OLP and 33 healthy controls. Each sample in each group was measured in triplicate and the experiment was repeated $\geq 3$ times. Data are presented as box-whisker plots; the boxes represent the interquartile region, the whiskers represent $95 \%$ confidence intervals and dots represent outliers. P-values are provided for miRNAs that exhibited a significant difference in expression in OLP patients compared with the Con group. miRNA, microRNA; miR-214, miRNA-214; OLP, oral lichen planus; Con, control.
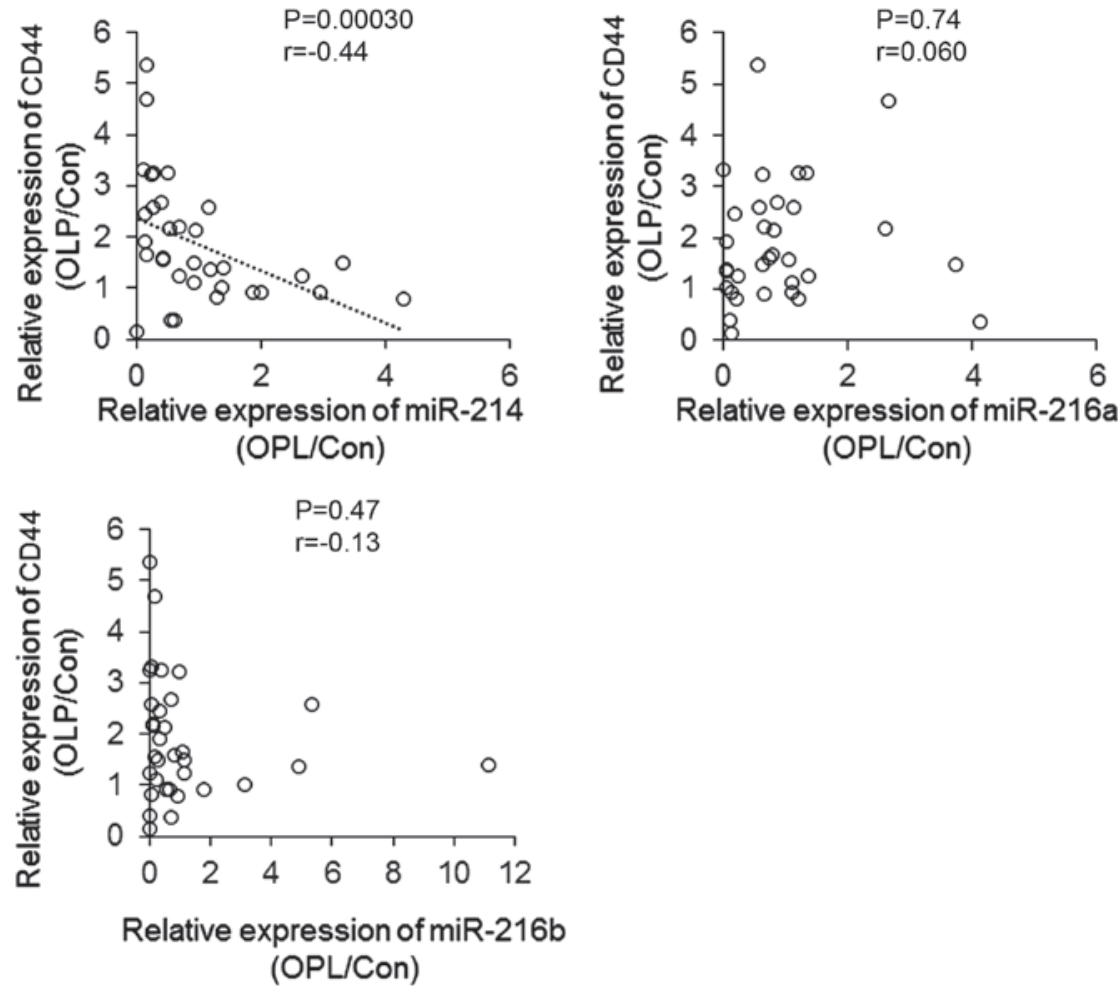

Figure 3. A negative correlation exists between the level of miR-214 and CD44. The expression of OPN and CD44 in mucosa samples was detected by western blot analysis and the results were quantified by Quantity One software. The association between CD44 protein level and the relative expression of CD44 was analyzed by correlation analysis. miR-214, microRNA-214; OPN, osteopontin; OLP, oral lichen planus; Con, control. 
A

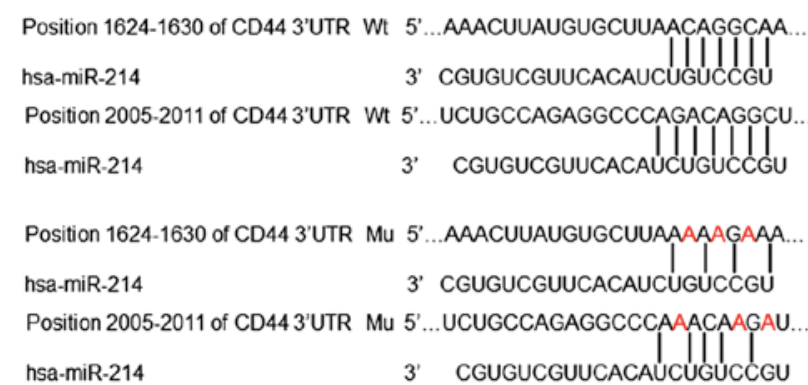

B

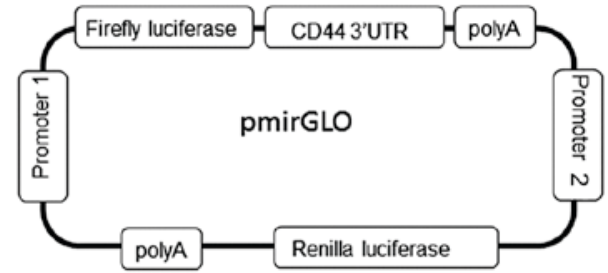

$\mathrm{D}$

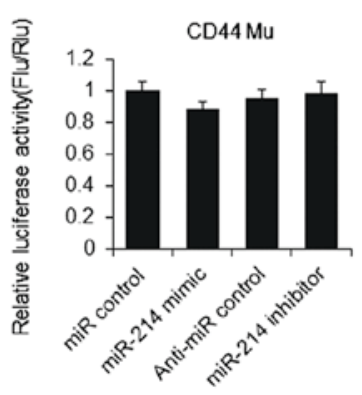

Figure 4. miR-214 represses CD44 expression by targeting the CD44 3'UTR. (A) Schematic diagram for predicted target sites of miR-214 and Mu vector construction. Red letters represent the mutant sites. (B) Schematic diagram for dual luciferase reporter vector construction. (C) HeLa cells were transfected with Wt reporter vector and miR-214 mimic or inhibitor for $48 \mathrm{~h}$. Each sample in each group was measured in triplicate and the experiment was repeated 3 times. (D) HeLa cells were transfected with Mu reporter vector and miR-214 mimic or inhibitor for $48 \mathrm{~h}$. Each sample in each group was measured in triplicate and the experiment was repeated 3 times. Data are presented as the mean \pm standard deviation. ${ }^{*} \mathrm{P}<0.05$ and ${ }^{* * *} \mathrm{P}<0.01$. miR-214, microRNA-214; UTR, untranslated region; Mu, mutant; Wt, wild-type; Flu, firefly luciferase; Rlu, Renilla luciferase.

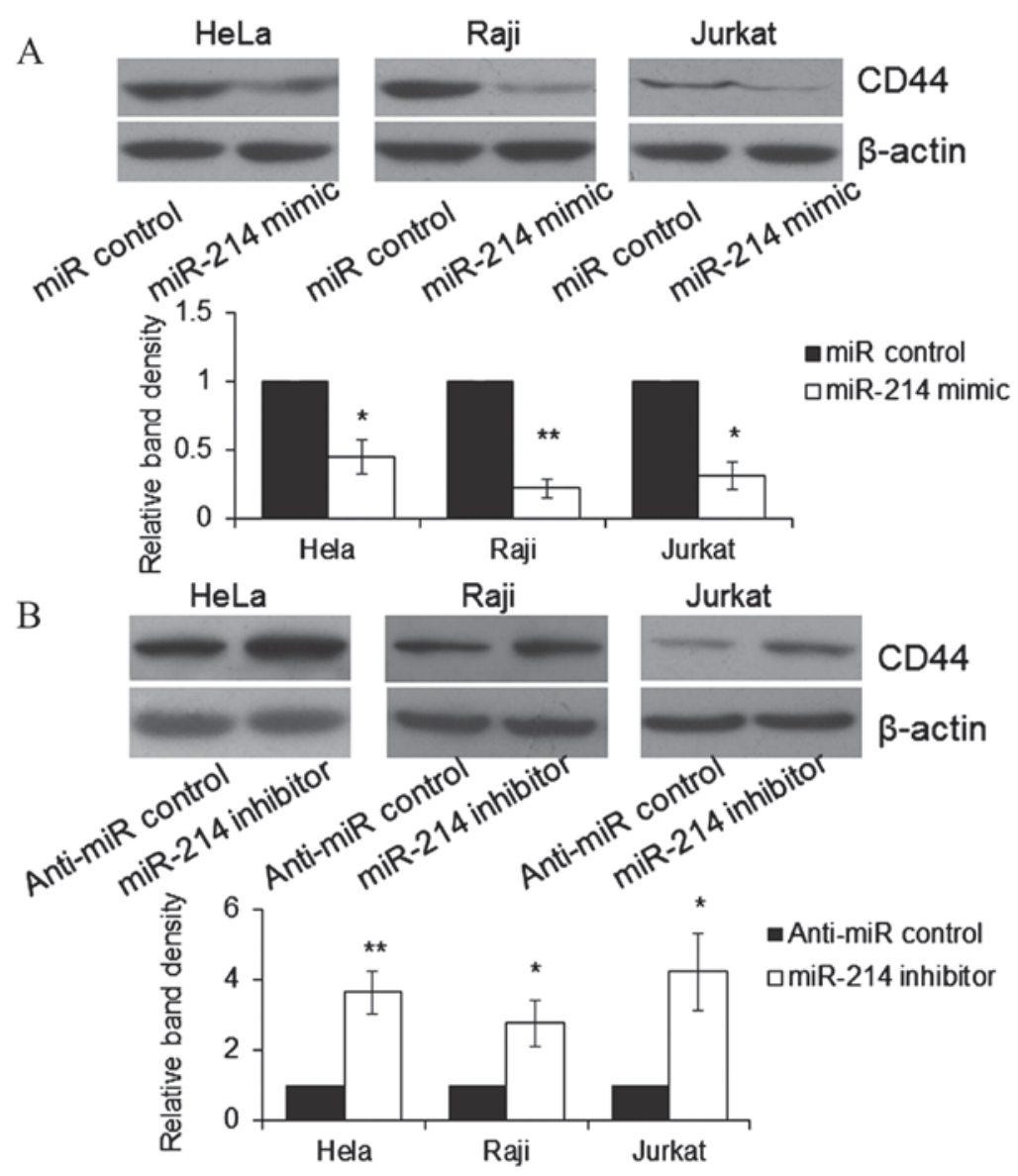

Figure 5. miR-214 represses the expression of endogenous CD44 in HeLa, Raji and Jurkat cells. miR-214 (A) mimic or (B) inhibitor was transfected into HeLa, Raji and Jurkat cells for $48 \mathrm{~h}$. Relative band density was determined by Quantity One software. Each sample in each group was measured in triplicate and the experiment was repeated $\geq 3$ times. Data are presented as the mean \pm standard deviation. ${ }^{*} \mathrm{P}<0.05$ and ${ }^{* * *} \mathrm{P}<0.01$ vs. control. miR-214, microRNA-214. 
to infiltrate the oral mucosa, in which the activated $\mathrm{T}$ cells trigger apoptosis of oral epithelial cells. Overexpression of OPN and CD44 was previously demonstrated to be present in the mucosa of patients with OLP, and it has been confirmed that OPN suppresses the apoptosis of activated $\mathrm{CD}^{+} \mathrm{T}$ cells via CD44 $(17,18)$. The present study initially confirmed the overexpression of OPN and CD44 in the mucosa of patients with OLP, compared with controls, by RT-qPCR and western blot analysis. As the upregulation of CD44 protein expression in OLP samples compared with controls, was greater than the observed increase in CD44 mRNA expression, and the fact that gene expression is regulated transcriptionally and post-transcriptionally, it was hypothesized that miRNAs may participate in the regulation of CD44 expression. By analyzing the association between CD44 protein level and relative miRNA expression, a strong negative correlation between the levels of miR-214 and CD44 in the mucosa samples of patients with OLP was demonstrated. Subsequently, the present study indicated that miR-214 represses endogenous CD44 expression by targeting the 3'UTR of CD44 in HeLa, Raji and Jurkat cells. The present study identified that reduced miR-214 is associated with OLP and miR-214 may be a potential candidate for drug development.

The present study demonstrated a direct association between miR-214 and CD44. However, the effect of reduced miR-214 expression on the survival of $\mathrm{T}$ cells requires further investigation. Furthermore, miR-214 has been previously identified as a tumor suppressor. Reports indicate that reduced miR-214 expression has been implicated in patients with ovarian, esophageal and pancreatic cancer (19-21). Furthermore, overexpression of miR-214 increased the drug sensitivity of cancer cells $(22,23)$. Therefore, reduced miR-214 expression may increase the risk of the carcinogenesis. The experiments in the present study were performed using biopsy samples and the identification of which specific cell types were involved in the observed alterations in expression was not possible. Therefore, further experiments, including laser capture microdissection, are required to determine which cell types have the most important role during the pathogenesis of OLP. In conclusion, the present study demonstrated a direct correlation between the expression of miR-214 and CD44 in mucosa samples of patients with OLP. In addition, the results confirmed CD44 as a direct target of miR-214. The current study identified that reduced miR-214 is associated with OLP and may be a candidate molecule for drug development.

\section{References}

1. Khan A, Farah CS, Savage NW, Walsh LJ, Harbrow DJ and Sugerman PB: Th1 cytokines in oral lichen planus. J Oral Pathol Med 32: 77-83, 2003

2. Gupta S and Jawanda MK: Oral Lichen Planus: An update on etiology, pathogenesis, clinical presentation, diagnosis and management. Indian J Dermatol 60: 222-229, 2015.

3. Dudhia BB, Dudhia SB, Patel PS and Jani YV: Oral lichen planus to oral lichenoid lesions: Evolution or revolution. J Oral Maxillofac Pathol 19: 364-370, 2015.
4. Ashkar S, Weber GF, Panoutsakopoulou V, Sanchirico ME, Jansson M, Zawaideh S, Rittling SR, Denhardt DT, Glimcher MJ and Cantor H: Eta-1 (osteopontin): An early component of type-1 (cell-mediated) immunity. Science 287: 860-864, 2000.

5. Weber GF, Ashkar S, Glimcher MJ and Cantor H: Receptor-ligand interaction between CD44 and osteopontin (Eta-1). Science 271: 509-512, 1996.

6. Chabas D, Baranzini SE, Mitchell D, Bernard CC, Rittling SR, Denhardt DT, Sobel RA, Lock C, Karpuj M, Pedotti R, et al: The influence of the proinflammatory cytokine, osteopontin, on autoimmune demyelinating disease. Science 294: 1731-1735, 2001.

7. Sakata M, Tsuruha JI, Masuko-Hongo K, Nakamura H, Matsui T, Sudo A, Nishioka K and Kato T: Autoantibodies to osteopontin in patients with osteoarthritis and rheumatoid arthritis. J Rheumatol 28: 1492-1495, 2001.

8. Chiocchetti A, Indelicato M, Bensi T, Mesturini R, Giordano M, Sametti S, Castelli L, Bottarel F, Mazzarino MC, Garbarini L, et al: High levels of osteopontin associated with polymorphisms in its gene are a risk factor for development of autoimmunity/lymphoproliferation. Blood 103: 1376-1382, 2004.

9. Zhou ZT, Wei BJ and Shi P: Osteopontin expression in oral lichen planus. J Oral Pathol Med 37: 94-98, 2008.

10. Santarelli A, Mascitti M, Rubini C, Bambini F, Zizzi A, Offidani A, Ganzetti G, Laino L, Cicciù $M$ and Lo Muzio L: Active inflammatory biomarkers in oral lichen planus. Int $\mathrm{J}$ Immunopathol Pharmacol 28: 562-568, 2015.

11. Friedman RC, Farh KK, Burge CB and Bartel DP: Most mammalian mRNAs are conserved targets of microRNAs. Genome Rese 19: 92-105, 2009

12. Gassling V, Hampe J, Açil Y, Braesen JH, Wiltfang J and Häsler R: Disease-associated miRNA-mRNA networks in oral lichen planus. PLoS One 8: e63015, 2013.

13. Livak KJ and Schmittgen TD: Analysis of relative gene expression data using real-time quantitative PCR and the 2(-Delta Delta C(T)) method. Methods 25: 402-408, 2001.

14. van der Waal I, Schepman KP and van der Meij EH: A modified classification and staging system for oral leukoplakia. Oral Oncol 36: 264-266, 2000.

15. Fischer AH, Jacobson KA, Rose J and Zeller R: Hematoxylin and eosin staining of tissue and cell sections. CSH Protoc 2008: pdb prot4986, 2008.

16. Chanmee T, Ontong P, Kimata K and Itano N: Key roles of Hyaluronan and its CD44 receptor in the stemness and survival of cancer stem cells. Front Oncol 5: 180, 2015.

17. Chaiyarit P, Thongprasom K, Satayut S, Dhanuthai K, Piboonratanakit P, Phothipakdee P, Subarnbhesaj A, Limlertmongkol S and Chaimusig M: Alteration of the expression of CD44 [corrected] isoforms in oral epithelia and saliva from patients with oral lichen planus. J Clin Immunol 28: 26-34, 2008.

18. Liu GX, Sun JT, Yang MX, Qi XM, Shao QQ, Xie Q, Qu X, Wei FC and Sun SZ: OPN promotes survival of activated T cells by up-regulating CD44 in patients with oral lichen planus. Clin Immunol 138: 291-298, 2011.

19. Kuninty PR, Bojmar L, Tjomsland V, Larsson M, Storm G, Östman A, Sandström P and Prakash J: MicroRNA-199a and -214 as potential therapeutic targets in pancreatic stellate cells in pancreatic tumor. Oncotarget 7: 16396-16408, 2016.

20. Liu Y, Zhou H, Ma L, Hou Y, Pan J, Sun C, Yang Y and Zhang J: MiR-214 suppressed ovarian cancer and negatively regulated semaphorin 4D. Tumour Biol 37: 8239-8248, 2016.

21. Xu Y and Lu S: Regulation of $\beta$-catenin-mediated esophageal cancer growth and invasion by miR-214. Am J Transl Res 7: 2316-2325, 2015.

22. Yu X, Luo A, Liu Y, Wang S, Li Y, Shi W, Liu Z and Qu X: MiR-214 increases the sensitivity of breast cancer cells to tamoxifen and fulvestrant through inhibition of autophagy. Mol Cancer 14: 208, 2015.

23. Phatak P, Byrnes KA, Mansour D, Liu L, Cao S, Li R, Rao JN, Turner DJ, Wang JY and Donahue JM: Overexpression of miR-214-3p in esophageal squamous cancer cells enhances sensitivity to cisplatin by targeting survivin directly and indirectly through CUG-BP1. Oncogene 35: 2087-2097, 2016. 